Rheumatology, Pavia, Italy; ${ }^{2}$ University of Genoa and Ospedale Policlinico San Martino, Internal Medicine, Genova, Italy; ${ }^{3}$ University of Pavia, PhD in Experimental Medicine, Pavia, Italy; ${ }^{4}$ National Referral Center for Rare Systemic and Autoimmune Diseases, Hôpital Cochin, Internal Medicine, Paris, France; ${ }^{5}$ Paris Descartes University, Paris 5, Paris, France

Background: Granulomatosis with polyangiitis (GPA) and microscopic polyangiitis (MPA) require glucocorticoids (GCs) and immunosuppressants (IS) to induce and maintain remission. At the era of highly active drugs and treat-to-target strategies, defining the goal to achieve in terms of remission could be beneficial for the long-term management.

Objectives: To assess the impact of prolonged remission or low disease activity state (LDAS) in GPA and MPA patients and its relationship with damage accrual.

Methods: Patients diagnosed with GPA and MPA, according to ACR criteria and/or Chapel Hill definitions, seen in two vasculitis centers and followed-up for $\geq 5$ years were included. Disease activity was assessed by BVAS, and damage accrual by the VDI. Three levels of remission were defined: complete remission (CR): BVAS $=0$ and negative ANCA in GCs-free and IS-free patients; clinical remission off therapy: no disease activity and positive ANCA in GCs-free and IS-free patients; clinical remission on therapy: no disease activity in patients with low dose GCs $(\leq 5 \mathrm{mg} / \mathrm{d})$ and/or IS. LDAS was defined as $0<B V A S \leq 3$ without major organ activity, no new disease activity, low-dose GCs ( $\leq 7.5 \mathrm{mg} /$ day) and well-tolerated IS. We defined remission or LDAS as prolonged when lasting $\geq 2$ consecutive years. The effect of prolonged remission and LDAS on damage accrual was evaluated.

Results: 167 patients were included: 128 (76.6\%) GPA, mean age $51.0 \pm 16.7$ years. At 5-years, mean VDI was 2.7 \pm 2.0 , mainly because of AAV-related items $(2.0 \pm 1.7)$ rather than treatment-related items $(0.7 \pm 1.0)$. During the 5-year follow-up, $10(6.0 \%)$ patients achieved prolonged CR, 6 (3.6\%) prolonged clinical remission off therapy, $89(53.3 \%)$ prolonged clinical remission on therapy, $42(25.1 \%)$ prolonged LDAS and $20(12.0 \%)$ never achieved LDAS. Damage accrual at 5-years in patients with prolonged $\mathrm{CR}$, clinical remission off therapy, clinical remission on therapy, LDAS or those never achieved LDAS was $1.6 \pm 1.1,1.8 \pm 1.7,2.3 \pm 1.9,3.8 \pm 2.0$ and $3.3 \pm 2.0$, respectively $(P<0.0001)$. Damage was comparable between patients in prolonged remission off therapy and those in remission on therapy $(P=0.3)$. In contrast, patients in prolonged LDAS or those never in LDAS had significantly more damage accrual $(P<0.0001$ and $P=0.01$, respectively) than those in prolonged remission off therapy. Eighty-one patients (49\%) reached a VDI $\geq 3$ at 5 -years. The inability to achieve prolonged remission was associated with a VDI $\geq 3$ at 5 -years (OR $5.07,95 \% \mathrm{Cl}$ 2.53-9.84, $\mathrm{P}<0.0001$ ), and considering only prolonged $\mathrm{CR}$ or clinical remission off therapy did not had any benefit on damage accrual. In contrast, achieving prolonged LDAS had no benefit compared to spending no time in LDAS $(P>0.99)$. Compared to patients achieving prolonged remission, those not able to achieve prolonged remission were younger ( $46 \pm 16.0$ vs. $53.5 \pm 16.6, P=0.001)$, had more frequent GPA $(\mathrm{P}=0.0003)$, had more $\mathrm{PR} 3-\mathrm{ANCA}(\mathrm{P}=0.006)$, had more ENT and lung involvement $(P<0.0001$ and $P=0.036$, respectively).

Conclusion: Sixty percent of GPA and MPA patients achieved prolonged remission, which was associated with a better outcome in terms of damage accrual. In contrast, prolonged LDAS was associated with increased damage and was not a sufficient target to achieve in GPA and MPA.

Disclosure of Interests: : None declared

DOI: 10.1136/annrheumdis-2020-eular.3101

\section{AB0468 AGE RELATED DIFFERENCES IN PATIENTS WITH GIANT CELL ARTERITIS}

P. Dieguez Pena ${ }^{1}$, B. Gimena Reyes ${ }^{1}$, B. Maure Noia ${ }^{1}$, A. Argibay ${ }^{1}$, C. Vázquez Triñanes ${ }^{1}$, A. J. Rivera Gallego ${ }^{1}$, A. Sousa Dominguez ${ }^{1}$, M. Estevez ${ }^{1}{ }^{1}$ Complejo Hospitalario Universitario de Vigo, Vigo, Spain

Background: Few data are available on the epidemiology and management of giant cell arteritis (GCA) in patients over 75 years despite the progressive aging in our societies. In other diseases this subgroup of patients presents important differences in the management and prognosis of their pathologies.

Objectives: To explore this situation by comparing two subgroups of patients older and younger than 75 , assesing possible changes in demographic characteristics, diagnostic tests, treatment and outcome.

Methods: We perform a retrospective review of charts, laboratory data, image studies, treatment and outcome of biopsy-proven GCA in our institution (Complejo Hospitalario Universitario de Vigo) between 1 January 2000 and 30 November 2019. Results: During study period 124 patients were analysed, 51 in the subgroup of $<75$ (mean age 64.6 (56-75)) and 73 in the subgroup of $>75$ (mean age 81.8 (76$89)$ ). There were no differences about sex (female $76.6 \%$ vs. $65 \%$ ) or in the Charlson index between the two groups (0-1 in $72 \%$ of patients). Older patients present more frequently with headache $(49.2 \%$ vs $32.3 \%)$, polymyalgia rheumatica $(53.4 \%$ vs $45.1 \%$ ), weight loss (48\% vs $39.2 \%)$ and ischaemic manifestations $(72.6 \%$ vs $51 \%)$, including visual disturbances (26\% vs $11.8 \%)$. Younger patients present more frequently with fever (33.3\% vs $19.2 \%$ ). Median ESR was similar: 98 vs $96 \mathrm{~mm} / \mathrm{h}$; median CPR was slightly higher in younger patients $94.5 \mathrm{mg} / \mathrm{dL}$ vs $71.59 \mathrm{mg} / \mathrm{dL}$.
PET-TC was performed more frequently at diagnosis in the subgroup of younger patients $(29.4 \%$ vs $12.3 \%)$ and during follow-up period in the other subgroup $(3.4 \%$ vs $7 \%$ ) with evidence of involvement of large vessels in 14 of them. Initial treatment consisted of corticosteroids in $100 \%$ of patients with the most frequent doses, in both groups, between $40-60 \mathrm{mg} /$ day of prednisone or equivalent. The subgroup of $<75$ were treated more aggressively receiving pulses of methylprednisolone $(125-250 \mathrm{mg})$ 12 patients $(23.5 \%)$, while in the subgroup $>75$ lower doses were started more frequently ( $<40 \mathrm{mg} /$ day in 21 patients, $28.7 \%$ ). Lowering corticosteroids to $<5 \mathrm{mg} /$ day were slower in the subgroup of patients $<75$ (47.1\% within the first 12 months) with respect to the $>75(58 \%)$. During the follow-up period 47 patients had at least one relapse, we did not observe statistical differences between both groups ( 21 patients $<75$ and 26 patients $>75$ ). Time to first relapse was more frequent within the first year of treatment (12 and 16 patients respectively). We could not identified any facto related to relapses in our multivariate analysis. There was no significant differences between both groups about starting MTX (33.3\% and 38\%) on relapses. Only two patients started TCZ (one in each group). Twenty-nine patients died during follow-up period $(11.7 \%$ in $<75$ vs. $31.5 \%$ in $>75$ ), but none were related with GCA

Conclusion: 1. No differences were observed in sex, comorbidities (including cardiovascular risk factors) or laboratory markers between both groups. 2 . Younger patients presented with less frequent ischaemic symptoms; however we perform a more powerful treatment, both in doses and duration.

Disclosure of Interests: None declared

DOI: 10.1136/annrheumdis-2020-eular.6558

\section{AB0469 CLINICAL DISEASE MIGHT BE DIVIDED INTO TWO PHENOTYPES IN ANCA ASSOCIATED VASCULITIS RESULTS OF A CLUSTER ANALYSIS}

E. Durak Ediboglu ${ }^{1}$, Ö. Gerçik ${ }^{1}$, E. Bilgin ${ }^{2}$, D. Solmaz ${ }^{1}$, I. Ocal ${ }^{1}$, Z. Soypacacı ${ }^{1}$, H. Cinakli ${ }^{1}$, A. Saglam ${ }^{2}$, S. Kiraz ${ }^{2}$, O. Karadag ${ }^{2}$, S. Akar ${ }^{1} .{ }^{1}$ Izmır Katıp Celebi, Izmir, Turkey; ${ }^{2}$ Hacettepe, Ankara, Turkey

Background: One of the controversial matters in ANCA associated vasculitis is the definition of disease based on clinical characteristics since there is remarkable overlap between disease groups. For instance, single organ disease like renal limited vasculitis (RLV) is not take place most of the definitions or classification criteria. Objectives: The aim of this study to determine clinical subgroups that may incorporate different clinical phenotypes including RLV in AAV patients followed up in two tertiary centers.

Methods: Baseline clinical features of AAV patients were studied. To analyse our data and identify sub-groups of AAV patients with similar clinical characteristics, a two-step cluster analysis using log-likelihood distance measures was performed. For clustering we evaluated the following variables: gender, age at symptom onset, the presence of major organ involvement (renal, upper and lower respiratory tract, skin, joint, eye) and ANCA specificity.

Results: In total 165 (87 [53\%] male, age at diagnosis $51.6 \pm 15.2$ years) out of 238 (126 [53\%] male, age at diagnosis $51.3 \pm 15.6$ years) AAV patients included in the analysis. Some of the demographic and clinical characteristics were summarized in the table 1. There are two distinct cluster in AAV patients. Of $78 \%$ those AAV patients with MPO/pANCA, 56\% with renal involvement and $89 \%$ without ENT involvement were in Cluster 1. Of $77 \%$ those patients with PR3/cANCA, $89 \%$ with arthritis, $74 \%$ with eye involvement, $83 \%$ with skin, $91 \%$ with upper, and $60 \%$ with lower respiratory tract involvement and $92 \%$ of those without renal disease were in Cluster 2 Most of the (89\%) patients classified as MPA and all as RLV were repositioned in Cluster 1 and $74 \%$ of GPA and $64 \%$ of EGPA patients were in Cluster 2.

Table 1. Baseline characteristics of 165patients with AAV

\begin{tabular}{lc}
\hline Age at diagnosis, mean years $\pm \mathrm{SD},(\mathrm{n})$ & $51.6 \pm 15.2,163$ \\
Male, $\mathrm{n}(\%)$ & $87(52.7)$ \\
Labaratory results at diagnosis & \\
GFR $=<60, \mathrm{n}(\%)$ & $100 / 145(69)$ \\
MPO-ANCA or p-ANCA /Pr3-ANCA or c-ANCA & $68(41.2) / 97(58.8)$ \\
Variants of AAVn (\%) & $26(15.8)$ \\
MPA & $108(65.5)$ \\
GPA & $11(6.7)$ \\
EGPA & $20(12.1)$ \\
RLV & \\
Organ systems involved at diagnosis $\boldsymbol{n}(\%)$ & $24(14.5)$ \\
Cutaneous & $23(13.9)$ \\
Eye & $90(54.5)$ \\
Ear, nose and throat & $111(67.3)$ \\
Low respiratory tract & $5 / 163(3.1)$ \\
Cardiovascular system & $10 / 164(6.1)$ \\
Gastrointestinal system & $129(78.2)$ \\
Renal & $16(9.7)$ \\
Peripheral nervous system &
\end{tabular}

GFR: glomeruler filtration rate; ANCA: antineutrophil cytoplasmic antibodies; Pr3: protein ase 3; MPO: myeloperoxidase; p-ANCA: perinuclear ANCA; c-ANCA: cytoplasmic ANCA MPA: Microscopic Polyangiitis; GPA: Granulamatosis and Polyangiitis; EGPA:Eosinophilic Granulomatosis with Polyangiitis; RLV: Renal limited vasculitis 